\title{
Single nucleotide polymorphism-based microarray analysis for the diagnosis of hydatidiform moles
}

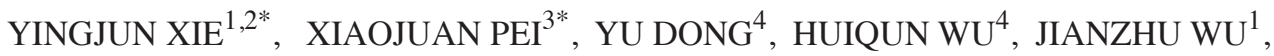 \\ HUIJUAN SHI ${ }^{4}$, XUYING ZHUANG ${ }^{5}$, XIAOFANG SUN ${ }^{2}$ and JIALING HE ${ }^{6}$ \\ ${ }^{1}$ Fetal Medicine Center, The First Affiliated Hospital of Sun Yat-sen University; \\ ${ }^{2}$ Key Laboratory for Major Obstetric Diseases of Guangdong Province, \\ Key Laboratory of Reproduction and Genetics of Guangdong Higher Education Institutes, \\ The Third Affiliated Hospital of Guangzhou Medical University, Guangzhou, Guangdong 510150; \\ ${ }^{3}$ Department of Pathology, The Huizhou Municipal Center People Hospital, Huizhou, Guangdong 516001; \\ ${ }^{4}$ Department of Pathology, The First Affiliated Hospital of Sun Yat-Sen University, Guangzhou, Guangdong 510080; \\ ${ }^{5}$ Tianjin Public Security Traffic Management Bureau, Tianjin 300241; ${ }^{6}$ Experimental Animal Center, \\ Research Institute for National Health and Family Planning Commission, \\ Beijing 100081, P.R. China
}

Received March 21, 2015; Accepted April 11, 2016

DOI: $10.3892 / \mathrm{mmr} .2016 .5211$

\begin{abstract}
In clinical diagnostics, single nucleotide polymorphism (SNP)-based microarray analysis enables the detection of copy number variations (CNVs), as well as copy number neutral regions, that are absent of heterozygosity throughout the genome. The aim of the present study was to evaluate the effectiveness and sensitivity of SNP-based microarray analysis in the diagnosis of hydatidiform mole (HM). By using whole-genome SNP microarray analysis, villous genotypes were detected, and the ploidy of villous tissue was determined to identify HMs. A total of 66 villous tissues and two twin tissues were assessed in the present study. Among these samples, 11 were triploid, one was tetraploid, 23 were abnormal aneuploidy, three were complete genome homozygosity, and the remaining ones were normal ploidy. The most noteworthy finding of the present study was the identification of six partial HMs and three complete HMs from those samples that were not identified as being HMs on the basis of the initial diagnosis of experienced obstetricians. This study has demonstrated that the application of an SNP-based microarray analysis was able to increase the sensitivity of diagnosis for HMs with partial
\end{abstract}

Correspondence to: Dr Huijuan Shi, Department of Pathology, The First Affiliated Hospital of Sun Yat-sen University, 58 Zhongshan No. 2 Road, Guangzhou, Guangdong 510080, P.R. China

E-mail: shihj@mail.sysu.edu.cn

*Contributed equally

Key words: single nucleotide polymorphism-based microarray, hydatidiform mole, villous tissue, spontaneous abortion and complete HMs, which makes the identification of these diseases at an early gestational age possible.

\section{Introduction}

Hydatidiform mole (HM) is an aberrant form of human pregnancy with no embryo, which has for a long time fascinated and puzzled scientists in equal measure, with an incidence rate of $\sim 1$ in 250 to 1 in 2,000 pregnancies (1-3). As persistent trophoblastic disease may develop from HM, it is the entity that has been morphologically investigated in the greatest detail, with specified morphological features $(4,5)$. In recent years, the management of early pregnancy has markedly improved, such that the majority of HMs present early on, as a failure of pregnancy or missed spontaneous abortion (SA) without the typical clinical signs of bleeding and hypertension (6). Based on the abnormal chromosomal patterns, HMs may be classified as complete HMs (CHMs) or partial HMs (PHMs) (7,8). Persistent gestational trophoblastic disease may develop in up to $15 \%$ of cases of CHMs, or in $0.5-1 \%$ cases of PHMs $(4,5)$. Therefore, the distinction of HMs from non-molar (NM) specimens, and their subclassification, is important in terms of clinical practice and investigative studies.

However, the histopathological division of spontaneously arrested pregnancies into HMs and NM SAs has consistently proven to be a challenge, since several research groups have noted a continuous variation in molar degeneration $(8,9)$. At an early gestational age, the overall sensitivity and positive predictive value of ultrasound diagnosis for HMs was $<50 \%$ in a study population (10). This challenge is currently more problematical, due to the early evacuation of arrested pregnancies based on ultrasonography, and prior to the manifestations of all the histopathological features. Therefore, a considerable number of HMs are only identifiable on the basis of further morphological and genetic investigations (9). 
Table I. Results of the single nucleotide polymorphism array for the 68 specimens.

\begin{tabular}{|c|c|c|c|}
\hline Parameter & Non-molar & $\mathrm{CHM}$ & PHM \\
\hline Number & 59 & 3 & 6 \\
\hline Complete homozygosity & & $3(46, \mathrm{XX})$ & \\
\hline Triploid & & & 3 \\
\hline \multirow[t]{9}{*}{ Additional abnormalities } & Trisomy $2(n=1)$ & & Trisomy $13(\mathrm{n}=1)$ \\
\hline & Trisomy $4(n=2)$ & & Trisomy $20(n=1)$ \\
\hline & Trisomy $9(\mathrm{n}=1)$ & & Tetraploidy $(\mathrm{n}=1)$ \\
\hline & Trisomy 15 (n=1) & & \\
\hline & Trisomy 16 (n=7) & & \\
\hline & Trisomy 18 (n=3) & & \\
\hline & Trisomy 22 (n=1) & & \\
\hline & Monsomy X (n=5) & & \\
\hline & 22q11.2 DS $(n=1)$ & & \\
\hline Cross-contamination & & & Fetal $(n=1)$ \\
\hline Total no. of loci with failed amplification & None & None & None \\
\hline
\end{tabular}

CHM, complete hydatidiform mole; DS, deletion syndrome; PHM, partial hydatidiform mole.

To date, various techniques, including chromosomal comparative genomic hybridization $(\mathrm{CGH})$, array (or microarray-based) $\mathrm{CGH}$, fluorescence in situ hybridization (FISH), multiplex ligation-dependent probe amplification (MLPA) and quantitative fluorescent polymerase chain reaction (QF-PCR), have circumvented several disadvantages inherent to conventional cytogenetic techniques, including poor chromosome preparations, culture failure and maternal cell contamination. As a result of these improvements on conventional cytogenetic techniques, more abnormalities have been identified in early miscarriages by means of novel techniques $(6,11-17)$. However, at present, the contribution these novel techniques make to resolving clinical problems remains poorly understood.

Currently, single nucleotide polymorphism (SNP)-based microarray analysis enables the detection of copy number variations (CNVs), as well as genotype information, at multiple polymorphic loci throughout the genome $(18,19)$. Although the clinical utility of CNV detection is well established, information derived from SNP-based microarray analysis has only been utilized in a constitutional cytogenetics laboratory setting until comparatively recently (12). In the present study, to the best of our knowledge for the first time, a successful application of an SNP-based array has been made in the detection of six partial moles and three complete moles from 68 early pregnancy tissue samples that were not initially diagnosed as HMs by experienced obstetricians. The findings of the present study suggest that SNP-based microarray may serve as a promising method for a more sensitive diagnosis of HMs at an earlier stage.

\section{Materials and methods}

Tissue samples. The study included 68 patients. The ages of the patients ranged from 26 to 37 years (mean, 33.4 years), and the gestational ages ranged from 9 to 19 weeks (mean, 12.3 weeks). All the tissue samples were first subjected to SNP-array detection to elucidate the cause of the early miscarriage, or twins with fetal abnormalities. Since the samples that had previously been identified as HMs were sent to the pathology department for further analysis, all these 68 samples subjected to the SNP-based microarray analysis had not been initially diagnosed to be HMs by experienced obstetricians on the basis of clinical pathological features, obstetric history and their clinical impression. The samples were divided into two parts: One for genetic detection, the other for fixing in $4 \%$ buffered formalin (Guangzhou Wexis Biotech Ltd., Guangzhou, China) for later pathological analysis. Samples and pictures from patients were obtained following informed consent. This study was approved by the ethics committee of The First Affiliated Hospital of Sun Yat-sen University (Guangzhou, China).

SNP microarray. Villi or fetal tissues were examined and dissected in order to remove any maternal deciduas or blood clots, and the samples were repeatedly washed in sterile phosphate-buffered saline solution to avoid maternal cell contamination. Genomic DNA was extracted from peripheral blood samples using a QIAquick ${ }^{\circledR}$ blood kit (Qiagen, Inc., Valencia, CA, USA). DNA concentrations were determined using a NanoDrop spectrophotometer (ND-1000, V.3.1.2; Nanodrop, Thermo Fisher Scientific, Inc., Wilmington, DE, USA). Subsequently, DNA samples (250 ng) were hybridized to an Affymetrix CytoScan ${ }^{\circledR} 750 \mathrm{~K}$ array kit (Affymetrix, Inc., Santa Clara, CA, USA), according to the manufacturer's protocol. This array has been developed specifically for cytogenetics research, and offers in excess of two million markers across the genome, including SNP probes and probes to detect CNVs (Cyto-arrays). CEL files, obtained by scanning CytoScan ${ }^{\circledR}$ arrays, were analyzed using Chromosome Analysis Suite software (NetAffx 33.1; Affymetrix, Inc.), using annotations of genome version GRCH37 (hg19). Gains and losses that affected a minimum of 50 markers of length $400 \mathrm{kbp}$ were initially considered. 
Chr.1

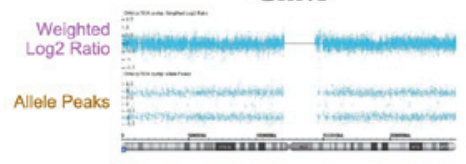

Chr.4

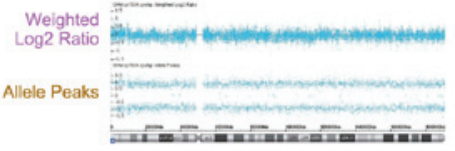

Chr.7

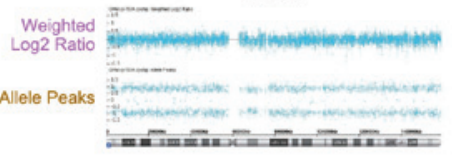

Chr.10

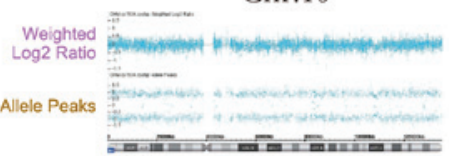

Chr.13

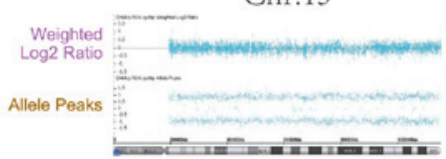

Chr.16

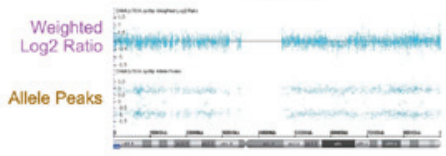

Chr.19

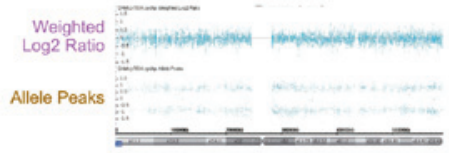

Chr.22

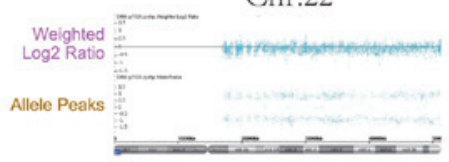

Chr.2

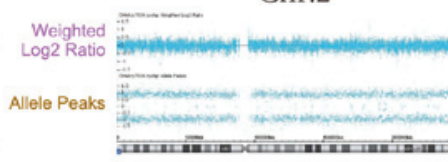

Chr.5

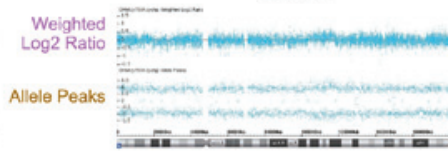

Chr.8

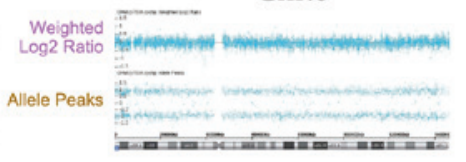

Chr.11

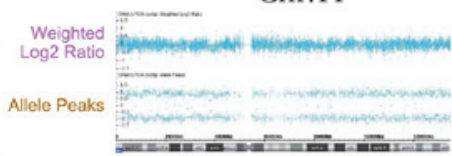

Chr.14

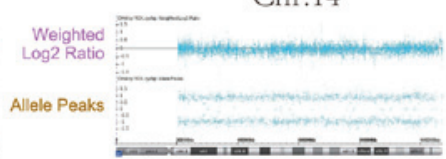

Chr.17

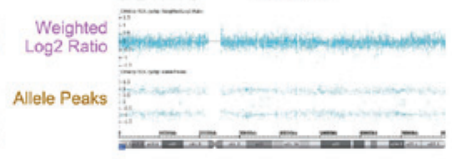

Chr.20

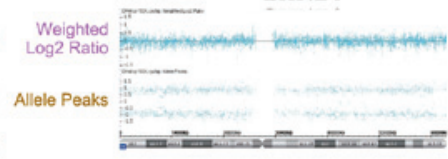

Chr.X

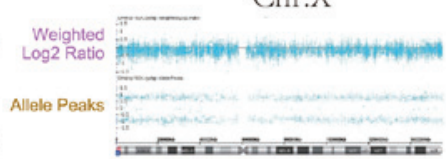

Chr.3

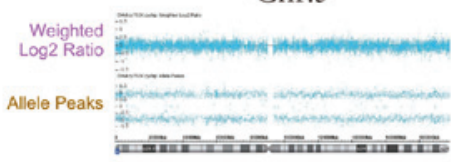

Chr.6

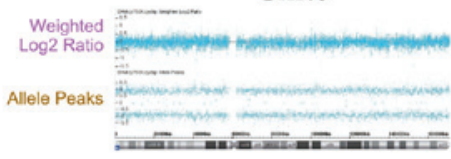

Chr.9

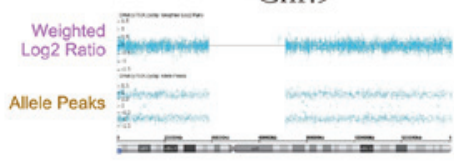

Chr.12

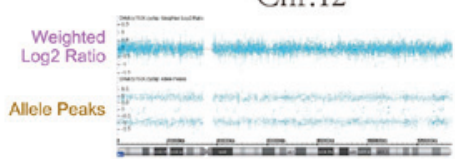

Chr.15

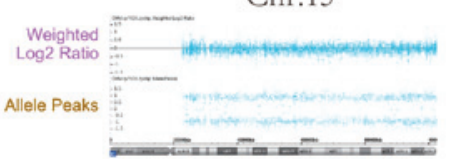

Chr.18

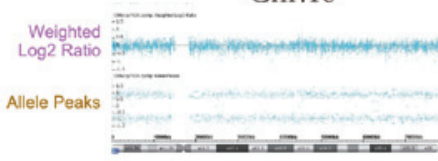

Chr.21

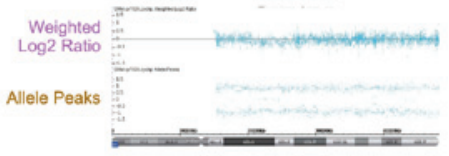

Chr.Y

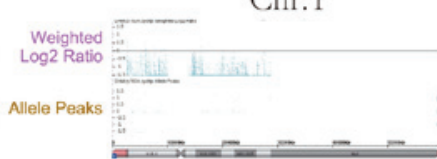

Figure 1. Single nucleotide polymorphism array on the entire chromosomal complement from one case of complete hydatidiform mole. Each chromosome was calculated by weighted $\log 2$ ratio, and presentation of the allele peak information revealed a large loss of heterozygosity, extending to the whole chromosome. Chr, chromosome.

Confirmatory test and FISH. The positive results identified by SNP-array detection were isolated, and the ploidy status was confirmed by FISH with probes corresponding to abnormal chromosomes (Abbott Molecular Inc., Des Plaines, IL, USA) analysis with the cells from the villous tissues, including four cases of triploid, and two cases of abnormal, aneuploidy Analysis was performed using a BX61 Olympus epifluorescence microscope (Olympus, Tokyo, Japan) with Applied Spectral Imaging FISH View 6.0 software (Applied Spectral Imaging, Inc., Carlsbad, CA,USA). Complete homozygosity was confirmed by molecular genotyping with short tandem repeat (STR) genetic markers, including three cases of CHM. A final diagnosis of HM was made on the basis of the subsequent pathological analysis and clinical follow-up information.

\section{Results}

SNP-array analysis detected nine HMs from 68 specimens. A total of 68 specimens, which were not initially diagnosed as HMs by the experienced obstetricians, were successfully detected by SNP-array analysis with consensus final pathological diagnoses of NMs and HMs obtained from the pathology archives (Table I). Among these samples, 11 were triploid, one was tetraploid, 23 were abnormal aneuploidy, three were complete genome homozygosity, and the remaining ones were 


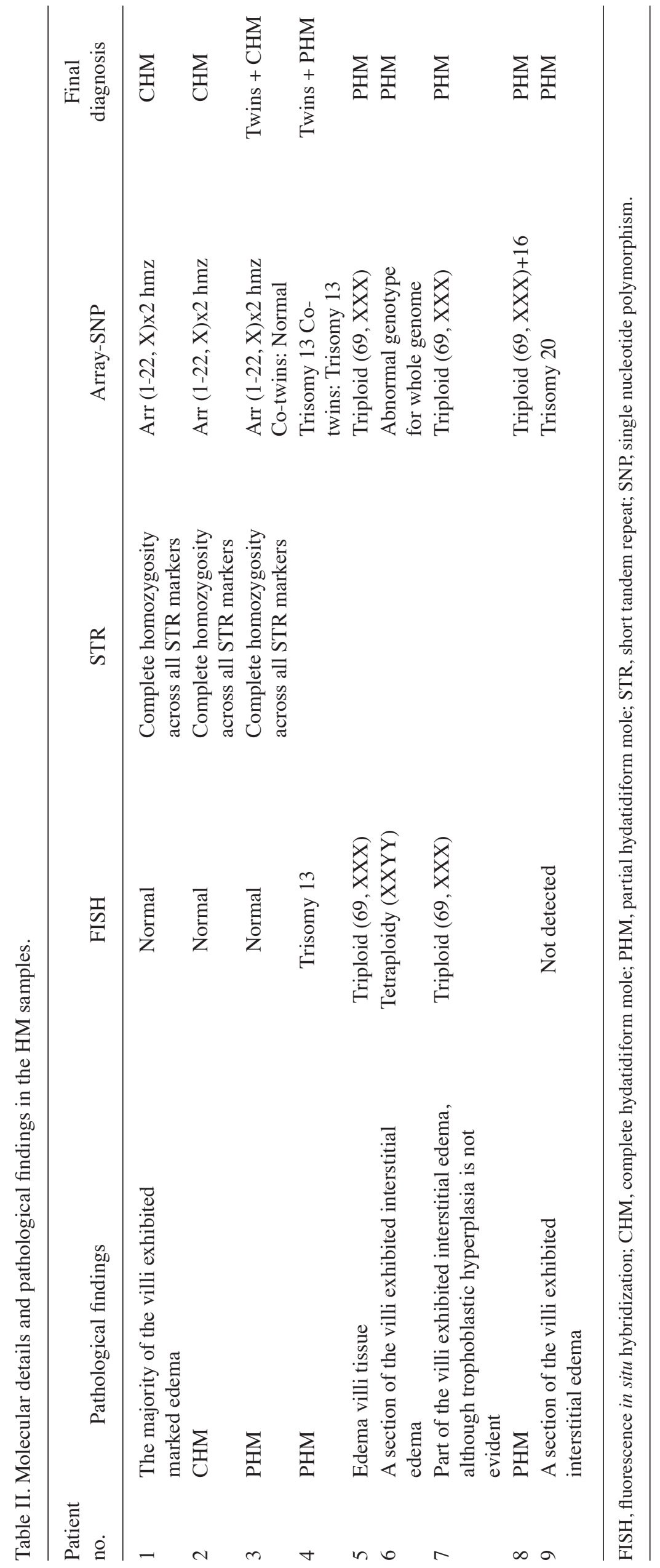


A

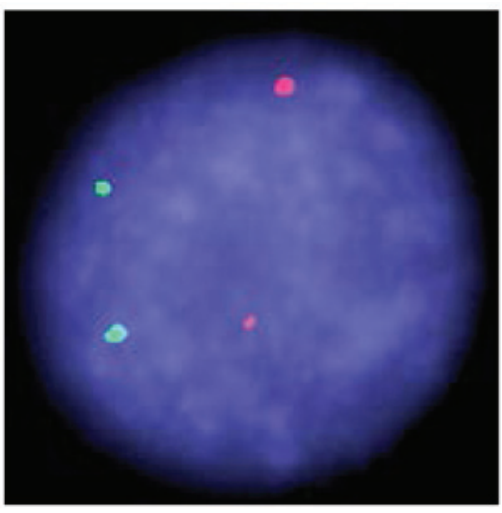

C

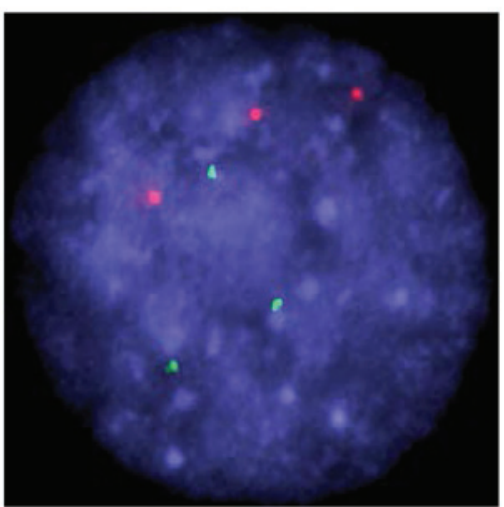

B

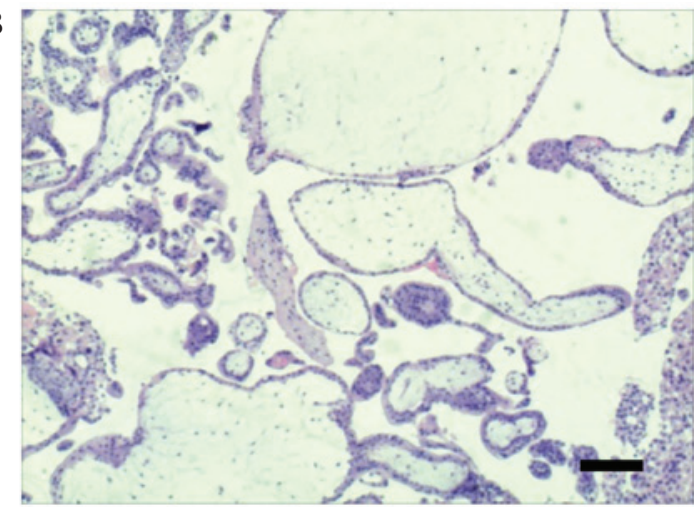

D

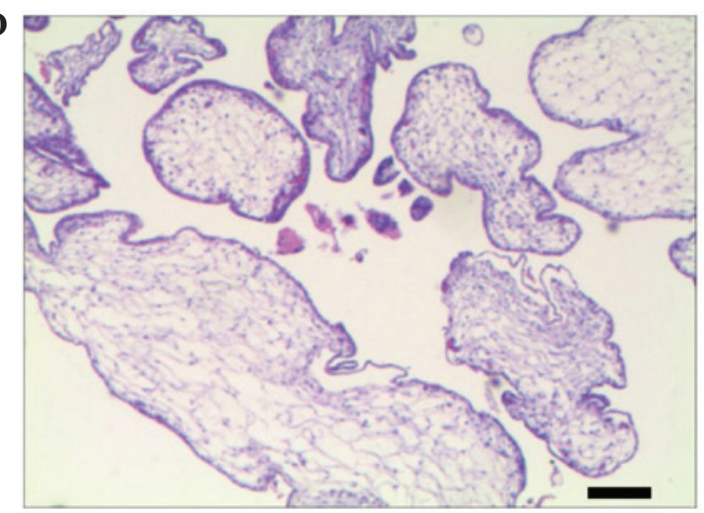

Figure 2. FISH and pathology sections, and microscopic sections and hematoxylin and eosin staining of the villous tissue, for two representative patients (nos. 3 and 4 in the present study). (A) FISH analysis with chromosome 13 (stained green) and 21 (stained red) for patient 3. (B) The microscopic section and hematoxylin and eosin staining of the villous tissue for patient 3 provides a representative image of complete hydatidiform mole. (C) FISH analysis of patient 4. (D) The microscopic section of patient 4 provides a representative image of partial hydatidiform mole. Scale bar=200 $\mu \mathrm{m}$. FISH, fluorescence in situ hybridization.

normal ploidy. Fig. 1 shows a representative SNP array on the entire chromosomal complement from one case of CHM.

Confirmatory test for nine HMs detected by SNP-array analysis. The performance of the SNP array was validated by clinical pathological tests involving microscope sections, FISH and molecular genotyping with STR markers for nine cases, which were determined to be HMs on performing SNP-array analysis. These results are summarized in Table II.

In the case of an unusual HM (patient no. 3: A 32-year-old primigravida woman at 19 weeks' gestation with one fetal mortality), the fetal heart stopped beating and the placenta was observed as a dark, liquid area of varying sizes, with the largest diameter of a liquid patch measuring $10 \mathrm{~mm}$, clinically consistent with a PHM. The serum human chorionic gonadotropin level at 19 weeks' gestation was measured as 1,692,348 $\mathrm{mIU} / \mathrm{ml}$. Dilatation and curettage were performed, yielding an anechoic sac containing edema, a placenta and a fetus with multiple malformations. Genotype analysis by FISH revealed normal ploid of chromosomes 13 (green) and 21 (red) for this sample (Fig. 2A), which was diagnosed as CHM by SNP array (Table II). Microscopic sections of the villous tissue revealed focal trophoblastic proliferation associated with two morphologically distinct populations of chorionic villi: Hydropic villi with central cisterns, and smaller, slightly irregular villi indicating a typical characteristic of CHM (Fig. 2B). Fig. 2C shows a representative FISH analysis for a triploid PHM sample, and Fig. 2D shows a representative section image of PHM (patient 4).

The microsatellite genotyping results for a case of CHM are shown in Fig. 3. A total of 21 STR loci contained in the identifier assay are shown for both the fetus and the mother villous samples. Each locus in the fetus was homozygous, as indicated by the presence of only one peak, and the absence of an association between the fetus and mother was evident in 13 loci (boxes). Complete homozygosity across all STR markers, in this case, was consistent with monospermy.

\section{Discussion}

Morphological features have been investigated in the greatest detail for HMs, which contribute towards early pregnancy loss to a substantial degree, and may also lead to the development of gestational trophoblastic disease $(2,4,6)$. In the present study, samples were assessed which were not initially diagnosed as HMs by experienced obstetricians, using SNP-array analysis to evaluate potential molar specimens. Our analysis led to the identification of nine cases of HM, including twins and single pregnancies; all these cases had an abnormal karyotype or genotype. In particular, three cases were identified of entire genome uniparental disomy, which would not have been identified using FISH, MLPA or cytogenetic studies. Trisomy was also identified in one of the twins that were diagnosed with PHM. In the remaining case, triploidy was identified with PHM. According to our results, a typical CHM exhibited 

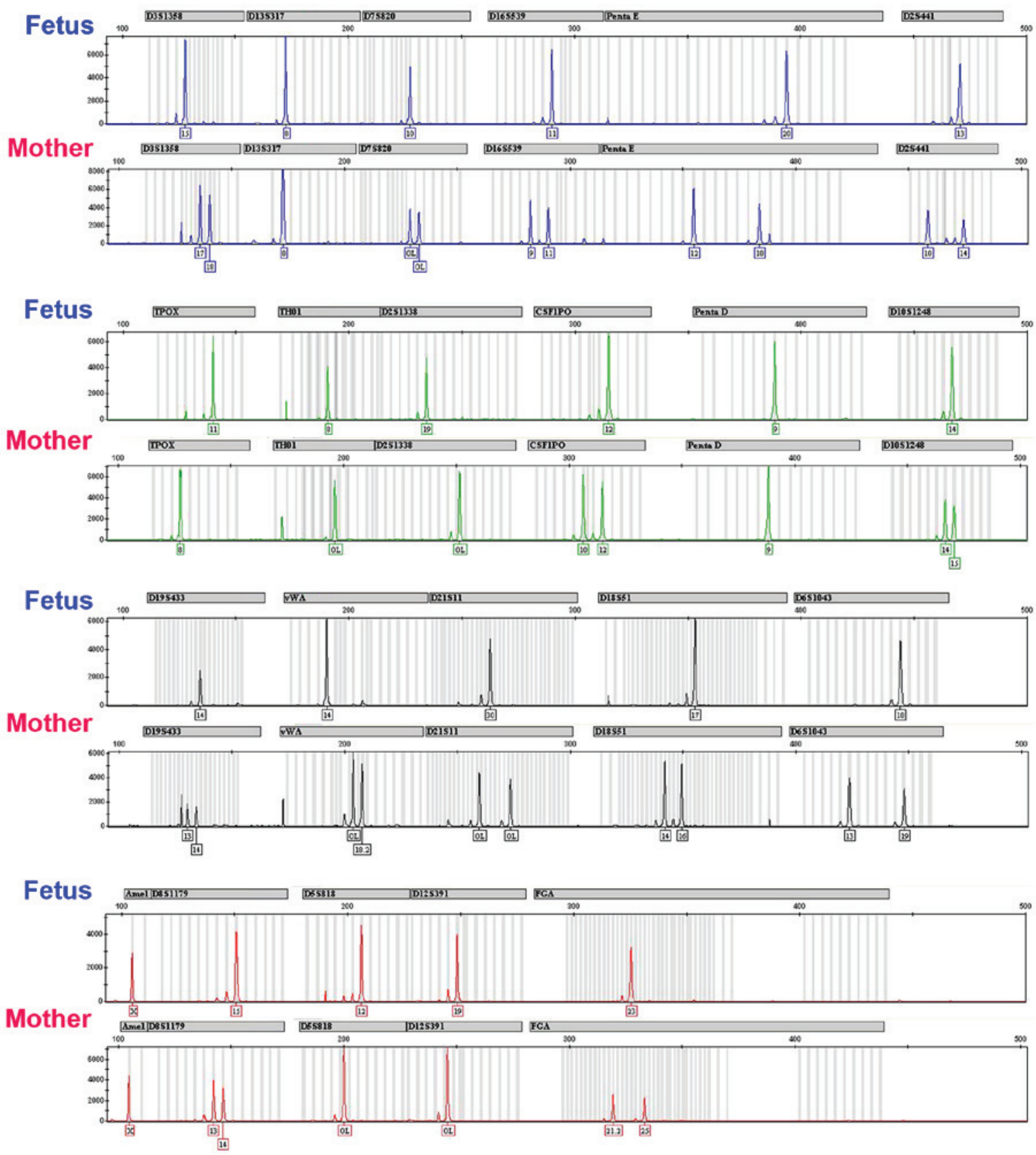

Figure 3. Microsatellite genotyping results for a case of CHM. A total of 21 short tandem repeat loci contained in the identifier assay are shown for the villous sample ('Fetus') and the mother. Each locus in the fetus was homozygous, as indicated by the presence of only one peak, and an absence of association between the fetus and mother was evident in 13 loci (illustrated by the boxes). Complete homozygosity across all short tandem repeat markers in this case was consistent with monospermy.

already distinct morphological features at a comparatively early gestational age $(20,21)$; however, in non-typical cases, the SNP array proved to be a valuable and reliable aid to the diagnosis.

Various techniques have been developed for the diagnosis of HMs; however, they all have certain limitations, which lead to a reduction in their accuracy. Pathological examination of the placental tissue is essential in the evaluation of miscarriage specimens, and a previous study used morphology in combination with flow cytometry to examine 150 missed abortion samples, with the determination of five PHMs (Table III) (22). However, morphological criteria alone had poor predictive power, unless combined with flow cytometry or other ploidy analytical approaches.
Polymorphic deletion probe (PDP) FISH probes, based on CNVs, are highly polymorphic and permit the in situ determination of genetic identity. In addition, FISH has the ability to identify specific chromosomal anomalies, including trisomy 13 , trisomy 16 , trisomy 21 and mosaicism, and is able to determine the sex chromosome complement. A previous study performed PDP FISH on diagnosed samples, including 13 CHMs, 13 PHMs, 13 NM abortions and an equivocal hydropic abortion (15). In this study, PDP FISH permitted the definitive diagnosis of CHMs in 5 of 13 cases for which the maternal and villous genotypes were mutually exclusive. Triploidy was observed in all PHMs, in which diandric triploidy was confirmed in six cases. In the equivocal hydropic abortion, PDP FISH, combined with p57 immunofluores- 
cence, revealed placental androgenetic/biparental mosaicism (Table III) (15). The ability to detect specific chromosomal abnormalities is dependent on the number of different FISH probes used. However, the cost of the FISH assay is also dependent on the number of FISH probes used (23).

PCR-based genomic fingerprinting with STR polymorphisms, which is the current gold standard molecular genetic technique used in identity testing (48 cases with 31 CHMs and 17 PHMs in 102 cases; Table III) is gaining in importance in confirming paternal origin HMs in investigative studies (14). STRs are repeated sequences of two or more nucleotides typically located in non-coding intron regions of the human genome. They are highly polymorphic, and are able to serve as robust markers of genetic identity (24). By identifying the genotypes of maternal and villous tissues at multiple STR loci, and determining the parental source of polymorphic alleles and their ratios in villous tissues, STR assays are useful in classifying molar pregnancies $(25,26)$. Several previous studies have demonstrated that STR genotyping may accurately distinguish between androgenetic diploid CHMs and biparental diploid $\mathrm{NMs}$, and between triploid diandric monogynic PHMs and triploid digynic monoandric NM products of conception (25-27). Since it is a PCR-based technique, STR analysis was limited by the insufficiency in DNA templates due to limited tissue sample, the requirement for pure populations of maternal and villous tissues, and the presence of maternal contamination in the interpretation of genotype data, which led to misclassification. In situ analysis SNP-arrays have the advantage of avoiding the technically challenging requirement of purifying maternal and villous tissues prior to analysis.

CGH, either alone or in combination with flow cytometry, has provided an alternative approach for the detection of chromosomal abnormalities in SAs $(13,28)$. CGH detects aneuploidy of all chromosomes, including partial monosomies or trisomies. However, it is not capable of reliably detecting changes in ploidy (since signal ratios are normalized over the whole chromosome complement); nor is it able to detect low-grade mosaicism or balanced structural anomalies (11). Since changes in ploidy and mosaicism are common among SAs, all normal CGH results must be followed up with flow cytometry or standard cytogenetic methods of diagnosis (29).

Compared with CGH, whole-genome SNP arrays have an important advantage in the detection of HMs. SNPs constitute comparatively small changes, usually involving the replacement of one nucleotide by another. Individual SNPs mark a unique genomic location, and are usually neutral in nature (30). Information provided by molecular karyotyping is directly associated with the physical and genetic map of the human genome. In addition to specific genotype data, analysis of SNP allele patterns is able to provide: i) Confirmation of CNV calls; ii) sensitivity for detection of mosaicism; and iii) detection of excessive homozygosity (12). For this reason, SNP arrays are more highly sensitive for the detection of HM.

Although the performance characteristics of SNP-array genotyping are superior to the majority of other methods for diagnosing HM, several limitations must be noted. SNP-array 
genotyping is able to detect triploidy, but not all cases of triploidy result in the phenotype of PHM (31). For this reason, it is important to combine SNP-array genotyping with clinical findings, histopathological features and genotyping results to ensure an accurate classification, for example, histology and p57 immunohistochemistry followed by microsatellite genotyping for equivocal cases $(14,15,27)$. Despite the aforementioned limitations to the clinical sensitivity and specificity, SNP-array genotyping is valuable since it yields direct information about the mechanism, even in the absence of the detection of the father's and mother's samples. The method is relatively inexpensive, and enables the identification of other genomic abnormalities that could potentially be misclassified as HMs on histopathological examination. Finally, and most importantly, in excluding a diagnosis of either CHM or PHM, SNP-array genotyping eliminates the requirement for clinical monitoring following pregnancy termination $(12,30,32)$.

Based on the characteristics of sensitivity, accuracy, efficiency and cost-effectiveness, the present results suggest that SNP arrays should provide a more useful strategy for the detection of HM specimens.

\section{Acknowledgements}

We would like to thank the staff of the prenatal center for supplying the patients' data. This study was supported by the National Natural Science Foundation of China (grant nos. 81201932 and 81372493$)$.

\section{References}

1. Steigrad SJ: Epidemiology of gestational trophoblastic diseases. Best Pract Res Clin Obstet Gynaecol 17: 837-847, 2003.

2. Benirschke K and Kaufmann P: Molar Pregnancies. In Pathology of the Human Placenta, Springer, New York, 653-685, 1995.

3. Benirschke $\mathrm{K}$ and Masliah E: The placenta in multiple pregnancy: Outstanding issues. Reprod Fertil Dev 13: 615-622, 2001.

4. Seckl MJ, Sebire NJ and Berkowitz RS: Gestational trophoblastic disease. Lancet 376: 717-729, 2010.

5. Seckl MJ, Fisher RA, Salerno G, Rees H, Paradinas FJ, Foskett M and Newlands ES: Choriocarcinoma and partial hydatidiform moles. Lancet 356: 36-39, 2000.

6. Sebire NJ and Rees H: Diagnosis of gestational trophoblastic disease in early pregnancy. Current Diagnostic Pathology 8: 430-440, 2002

7. Sebire NJ, Fisher RA and Rees HC: Histopathological diagnosis of partial and complete hydatidiform mole in the first trimester of pregnancy. Pediatr Dev Pathol 6: 69-77, 2003.

8. Szulman AE and Surti U: The syndromes of hydatidiform mole. II. Morphologic evolution of the complete and partial mole. Am J Obstet Gynecol 132: 20-27, 1978.

9. Vassilakos P, Riotton G and Kajii T: Hydatidiform mole: Two entities. A morphologic and cytogenetic study with some clinical consideration. Am J Obstet Gynecol 127: 167-170, 1977.

10. Kirk E, Papageorghiou AT, Condous G, Bottomley C and Bourne T: The accuracy of first trimester ultrasound in the diagnosis of hydatidiform mole. Ultrasound Obstet Gynecol 29: 70-75, 2007.

11. Zhang YX, Zhang YP, Gu Y, Guan FJ, Li SL, Xie JS, Shen Y, Wu BL, Ju W, Jenkins EC, et al: Genetic analysis of first-trimester miscarriages with a combination of cytogenetic karyotyping, microsatellite genotyping and arrayCGH. Clin Genet 75: 133-140, 2009.

12. Kearney HM, Kearney JB and Conlin LK: Diagnostic implications of excessive homozygosity detected by SNP-based microarrays: Consanguinity, uniparental disomy and recessive single-gene mutations. Clin Lab Med 31: 595-613, 2011.

13. Jobanputra V, Sobrino A, Kinney A, Kline J and Warburton D: Multiplex interphase FISH as a screen for common aneuploidies in spontaneous abortions. Hum Reprod 17: 1166-1170, 2002.
14. Furtado LV, Paxton CN, Jama MA, Tripp SR, Wilson AR, Lyon E, Jarboe EA, Thaker HM and Geiersbach KB: Diagnostic utility of microsatellite genotyping for molar pregnancy testing. Arch Pathol Lab Med 137: 55-63, 2013.

15. Chiang S, Fazlollahi L, Nguyen A, Betensky RA, Roberts DJ and Iafrate AJ: Diagnosis of hydatidiform moles by polymorphic deletion probe fluorescence in situ hybridization. J Mol Diagn 13: 406-415, 2011.

16. Menten B, Swerts K, Delle Chiaie B, Janssens S, Buysse K, Philippé J and Speleman F: Array comparative genomic hybridization and flow cytometry analysis of spontaneous abortions and mors in utero samples. BMC Med Genet 10: 89, 2009.

17. Robberecht C, Schuddinck V, Fryns JP and Vermeesch JR: Diagnosis of miscarriages by molecular karyotyping: Benefits and pitfalls. Genet Med 11: 646-654, 2009.

18. Wheeler E, Huang N, Bochukova EG, Keogh JM, Lindsay S, Garg S, Henning E, Blackburn H, Loos RJ, Wareham NJ, et al: Genome-wide SNP and CNV analysis identifies common and low-frequency variants associated with severe early-onset obesity. Nat Genet 45: 513-517, 2013.

19. Winchester L and Ragoussis J: Algorithm implementation for CNV discovery using Affymetrix and Illumina SNP array data. Methods Mol Biol 838: 291-310, 2012.

20. Fowler DJ,Lindsay I, Seckl MJ and Sebire NJ: Histomorphometric features of hydatidiform moles in early pregnancy: Relationship to detectability by ultrasound examination. Ultrasound Obstet Gynecol 29: 76-80, 2007.

21. Fowler DJ, Lindsay I, Seckl MJ and Sebire NJ: Routine pre-evacuation ultrasound diagnosis of hydatidiform mole: Experience of more than 1000 cases from a regional referral center. Ultrasound Obstet Gynecol 27: 56-60, 2006.

22. Grinschgl I, Mannweiler S, Holzapfel-Bauer M, Pferschy U, Hoefler $\mathrm{G}$ and Guertl B: The role of morphology in combination with ploidy analysis in characterizing early gestational abortion. Virchows Arch 462: 175-182, 2013.

23. Kipp BR, Ketterling RP, Oberg TN, Cousin MA, Plagge AM, Wiktor AE, Ihrke JM, Meyers CH, Morice WG, Halling KC and Clayton AC: Comparison of fluorescence in situ hybridization, p57 immunostaining, flow cytometry and digital image analysis for diagnosing molar and nonmolar products of conception. Am J Clin Pathol 133: 196-204, 2010.

24. Butler JM: Short tandem repeat typing technologies used in human identity testing. BioTechniques 43: ii-v, 2007.

25. Murphy KM, McConnell TG, Hafez MJ, Vang R and Ronnett BM: Molecular genotyping of hydatidiform moles: Analytic validation of a multiplex short tandem repeat assay. J Mol Diagn 11: 598-605, 2009.

26. Popiolek DA, Yee H, Mittal K, Chiriboga L, Prinz MK, Caragine TA and Budimlija ZM: Multiplex short tandem repeat DNA analysis confirms the accuracy of p57 (KIP2) immunostaining in the diagnosis of complete hydatidiform mole. Human Pathol 37: 1426-1434, 2006.

27. McConnell TG, Murphy KM, Hafez M, Vang R and Ronnett BM: Diagnosis and subclassification of hydatidiform moles using p57 immunohistochemistry and molecular genotyping: Validation and prospective analysis in routine and consultation practice settings with development of an algorithmic approach. Am J Surg Pathol 33: 805-817, 2009.

28. Daniely M, Aviram-Goldring A, Barkai G and Goldman B: Detection of chromosomal aberration in fetuses arising from recurrent spontaneous abortion by comparative genomic hybridization. Human Reprod 13: 805-809, 1998.

29. Lomax B, Tang S, Separovic E, Phillips D, Hillard E, Thomson T and Kalousek DK: Comparative genomic hybridization in combination with flow cytometry improves results of cytogenetic analysis of spontaneous abortions. Am J Hum Genet 66: 1516-1521, 2000.

30. Schwartz S: Clinical utility of single nucleotide polymorphism arrays. Clin Lab Med 31: 581-594, 2011.

31. Zaragoza MV, Surti U, Redline RW, Millie E, Chakravarti A and Hassold TJ: Parental origin and phenotype of triploidy in spontaneous abortions: Predominance of diandry and association with the partial hydatidiform mole. Am J Hum Genet 66: $1807-1820,2000$.

32. Conlin LK, Thiel BD, Bonnemann CG, Medne L, Ernst LM, Zackai EH, Deardorff MA, Krantz ID, Hakonarson $\mathrm{H}$ and Spinner NB: Mechanisms of mosaicism, chimerism and uniparental disomy identified by single nucleotide polymorphism array analysis. Hum Mol Genet 19: 1263-1275, 2010. 